\title{
Hipertensión portal secundaria a sarcoidosis hepática
}

\author{
Sofía G Rivarola, ${ }^{1}$ Mariano N Veltri, ${ }^{1}$ Ignacio Saguier Padilla, ${ }^{1}$ Alejandra Avagnina, ${ }^{2}$ Mariano \\ Biagioni, ${ }^{3}$ Alejo A Pérez De La Hoz, ${ }^{1}$ Patricia Vega, ${ }^{1}$ Andrea A Pisarevsky ${ }^{1}$
}

\author{
${ }^{1}$ Servicio de Clínica Médica VI, Cátedra del Hospital de Clínicas José de San Martín, Universidad de Buenos Aires. \\ ${ }^{2}$ Servicio de Anatomía Patológica, Hospital de Clínicas José de San Martín, Universidad de Buenos Aires. \\ ${ }^{3}$ Servicio de Hepatología del Hospital de Clínicas José de San Martín, Universidad de Buenos Aires. \\ Ciudad Autónoma de Buenos Aires, Argentina.
}

Acta Gastroenterol Latinoam 2020;50(1):45-49

Recibido: 28/09/2017 / Aceptado: 10/07/2019 / Publicado online: 23/03/2020 / https://doi.org/10.52787/tdlw1361

\section{Resumen}

La etiología de los granulomas hepáticos es variable dependiendo de la epidemiología. En países desarrollados la colangitis biliar primaria es la principal causa, seguida de la sarcoidosis, y en los paises en desarrollo, es la tuberculosis. Presentamos el caso de una paciente con hepatopatía crónica, cuyo diagnóstico anatomopatológico fue compatible con sarcoidosis. Se trataba de una mujer de 55 años, con antecedentes de diabetes tipo 2 insulino requiriente y sobrepeso. Su enfermedad debutó con sindrome ascítico edematoso, presentando posteriormente hemorragia digestiva alta de origen variceal. Fue derivada a nuestra institución para estudio. En el examen físico presentaba hepatoesplenomegalia y en el laboratorio FAL y yGT aumentadas con anti LKM y AMA no reactivos. La ecografía de abdomen evidenció hepatoesplenomegalia. La biopsia hepática in-

Correspondencia: Sofia G Rivarola

Avenida Córdoba 2351, piso 11 (sala 6), Hospital de Clínicas José de San Martín. Ciudad Autónoma de Buenos Aires, Argentina

Correo electrónico: sofiarivarolacalderon@gmail.com formó numerosos granulomas de ubicación portal. Enzima convertidora de angiotensina aumentada. Se diagnosticó sarcoidosis y se inició tratamiento con corticoides; se decidió iniciar deflazacort por presentar menor impacto en los niveles de glucemia. Evolucionó a los 12 meses con HDA que desencadenó su fallecimiento.

Palabras claves. Granuloma hepático, hipertensión portal, sarcoidosis.

\section{Portal Hypertension Secondary to Hepatic Sarcoidosis}

\section{Summary}

The etiology of hepatic granulomas varies depending on the epidemiology. In developed countries, primary biliary cholangitis (PBC) is the main cause followed by sarcoidosis. In developing countries, it is tuberculosis. We present the case of a patient with chronic liver disease, whose anatomopathological diagnosis was compatible with sarcoidosis. It was a 55-year-old woman, with a history of type 2 diabetes (DBT), insulin-requiring and overweight. Her disease debuted with SAE, subsequently presenting HDA of variceal origin. She was referred to our institution for study. Physical examination has hepatosplenomegaly and in the laboratory FAL and yGT augmented with non-reactive 
anti-LKM and AMA. Ultrasonography of the abdomen showed hepatosplenomegaly, without focal images. Hepatic biopsy puncture was performed, which reported numerous portal-site granulomas. The ECA dose was increased. The diagnosis of sarcoidosis was established and treatment with deflazacort was started. She presented an upper gastrointestinal bleeding causing her death.

Keywords. Hepatic granuloma, portal hypertension, sarcoidosis.

\section{Abreviaturas}

CBP: Colangitis biliar primaria.

DBT: Diabetes.

IMC: Indice de masa corporal.

SAE: Sindrome ascítico edematoso.

HDA: Hemorragia digestiva alta.

FAL: Fosfatasa alcalina.

yGT: Gamma glutamiltranspeptidasa.

LKM: Anticuerpos microsomales de higado y riñón.

AMA: Anticuerpo antimitocondrial.

ECA / ACE: Enzima convertidora de angiotensina /Angio tensin-converting enzyme.

TBC: Tuberculosis.

HTP: Hipertensión portal.

GASA: Gradiente de albúmina séricas-ascítico.

FAN: Anticuerpos antinucleares.

TAM: Tensión arterial media.

LPM: Latidos por minutos.

AST: Aspartato amino transferasa.

ALT: Alanina amino transferasa.

UI: Unidades internacionales.

$V N$ : Valor normal.

LDH: Lactato deshidrogenasa.

HbA1c: Hemoglobina glicosilada.

PAS: Tinción de ácido peryódico, reactivo de Schiff.

PPD: Prueba cutánea de derivado proteico purificadoreación de Mantoux.

HIV: Virus de inmunodeficiencia humana.

\section{Introducción}

La incidencia de granulomas en las biopsias hepáticas representa el 2 al 15\%. Su etiología es variable. En los países desarrollados, las causas más frecuentes son las no infecciosas, entre ellas la primera es la colangitis biliar primaria (CBP) y la segunda la sarcoidosis. En los países no desarrollados la tuberculosis es la principal causa, seguida de leishmaniasis visceral. ${ }^{1,2}$ Presentamos a continuación el caso de una paciente de mediana edad con hepatopatía crónica asociada a hipertensión portal, a la cual, mediante una biopsia hepática, se le diagnosticó sarcoidosis.

\section{Caso clínico}

Mujer de 55 años, con antecedentes de DBT tipo 2 insulino requiriente, hipertensión arterial, hipotiroidismo, colecistectomía en el año 1996 y sobrepeso. En febrero de 2015 presentó síndrome ascítico edematoso (SAE) con un gradiente albúmina sérica-líquido ascítico (GASA) compatible con hipertensión portal (HTP). Negaba consumo de alcohol o medicamentos. Las serologías virales para hepatitis $\mathrm{B}$ y $\mathrm{C}$ resultaron negativas. Los resultados de los autoanticuerpos fueron: FAN 1/160 moteado, ASMA 1/80, anti LKM y AMA no reactivo. A los 6 meses presentó hemorragia digestiva alta (HDA) secundaria a várices esofágicas, requiriendo reiteradas colocaciones de bandas. Fue derivada a nuestra institución para estudio.

A su ingreso la paciente se encontraba normotensa, TAM: $70 \mathrm{mmHg}$, frecuencia cardíaca de $60 \mathrm{LPM}$, temperatura corporal: $36^{\circ} \mathrm{C}$, saturando a $98 \%$ al aire ambiente. Lúcida, no presentaba flapping, reflejos osteotendinosos y pares craneales conservados. Sin signos de foco motor, sensitivo ni meníngeos. Refería prurito generalizado. Presentaba palidez cutánea mucosa, sin ictericia, con lesiones de rascado en región dorsal y en miembros inferiores. Ruidos cardíacos normofonéticos con silencios libres, pulsos periféricos conservados. Buena mecánica ventilatoria, murmullo vesicular conservado. Se palpaba tensión abdominal superficial aumentada, sin defensa ni reacción peritoneal, hígado palpable a $5 \mathrm{~cm}$ del reborde costal, no descendido, de consistencia firme, bazo palpable a $2 \mathrm{~cm}$ del reborde costal. En el laboratorio presentaba hematocrito de $31 \%$, leucocitos de $4.370 / \mathrm{mm}^{3}$, plaquetas de $83.000 / \mathrm{mm}^{3}$, uremia de $30 \mathrm{mg} / \mathrm{dl}$, creatinina de $0,78 \mathrm{mg} / \mathrm{dl}$, TP: $82 \%$, proteínas totales de $7,6 \mathrm{~g} / \mathrm{dl}$, albúmina de 3,2 $\mathrm{g} / \mathrm{dl}$, bilirrubina total de $0,8 \mathrm{mg} / \mathrm{dl}$, bilirrubina directa de $0,5 \mathrm{mg} / \mathrm{dl}$, AST de $22 \mathrm{UI} / \mathrm{L}$ (VN: hasta 37 UI/L), ALT de 21 UI/L (VN: hasta 40 UI/L), FAL de 336 UI/L (VN: 40-129 UI/L), GT 187 UI/L (VN: hasta $37 \mathrm{UI} / \mathrm{L}), \mathrm{LDH} 308 \mathrm{UI} / \mathrm{L}$, gammaglobulinas 2,46 g/dl (de tipo policlonal), HbA1c de 13,5\%. Ecografía de abdomen: hígado de forma conservada, tamaño aumentado, eco estructura heterogénea difusa de bordes lobulados, sin imágenes focales, con escasa cantidad de líquido libre, adenopatías en hilio hepático, esplénico, peripancreáticas y retroperitoneales, recanalización de la vena umbilical secundario a hipertensión portal. Se decidió realizar una 
punción biopsia hepática. La anatomía patológica mostró el parénquima hepático con numerosos granulomas de ubicación portal, compuestos por hepatocitos epitelioides y células gigantes multinucleadas sin necrosis central y con marcada hialinosis peri-granulomatosa. Se identificó, además, en el extremo de la biopsia, una extensa playa de fibrosis sin infiltrado inflamatorio. Se realizaron técnicas de PAS, Ziehl Neelsen y Grocott, que resultaron negativas para parásitos, bacterias y hongos. La inflamación granulomatosa fue vinculada en primer lugar a sarcoidosis (Figuras 1, 2 y 3). Se solicitó prueba de PPD, que resultó no reactiva, y una tomografía de tórax que mostró múltiples adenopatías mediastinales e hiliares bilaterales (22 mm), imágenes ganglionares en ambas regiones axilares, sin infiltrados patológicos pulmonares. El dosaje de enzima convertidora de angiotensina (ECA) fue de 95,4 UI/L (VN: 8-52 UI/L). Con los resultados obtenidos se estableció el diagnóstico de sarcoidosis y se inició tratamiento con deflazacort $50 \mathrm{mg}$ por día, ya que la paciente presentaba hiperglucemias y este corticoide tendría

Figura 1. Granulomas portales con necrosis caseosa (tinción con hematoxilina eosina 250X).

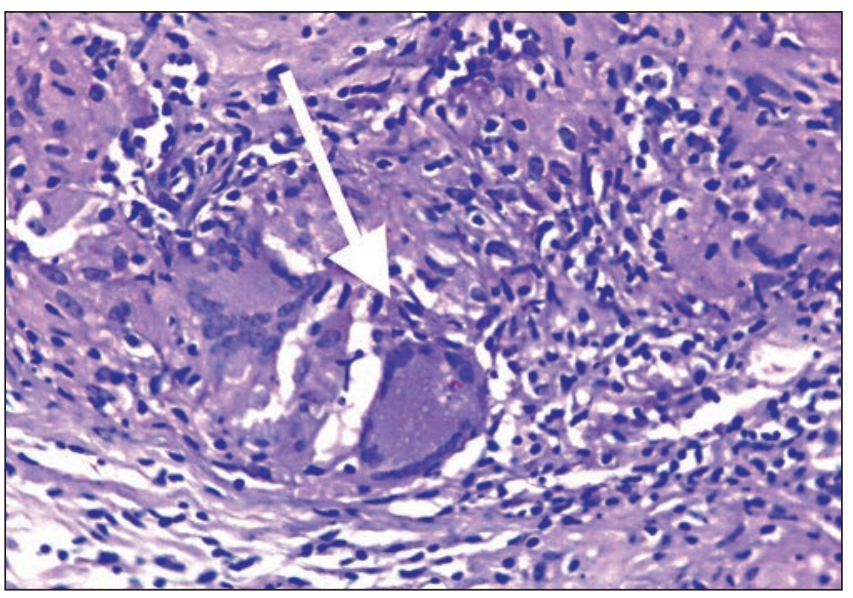

Figura 2. Marcada expansión portal por la presencia de granulomas no caseificantes (tinción con PAS. 100X).

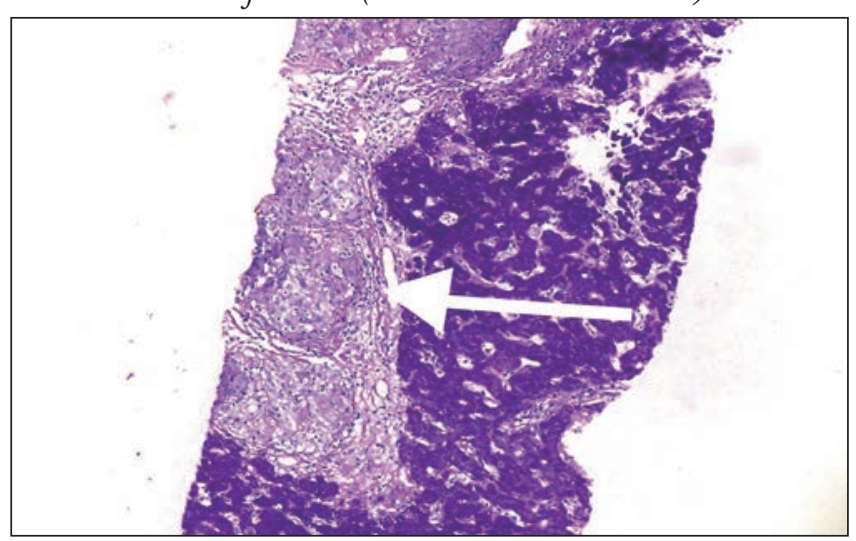

Figura 3. Fibrosis portal asociada a la presencia de granulomas sarcoidales (tinción con Tricrómico de Masson. 100X).

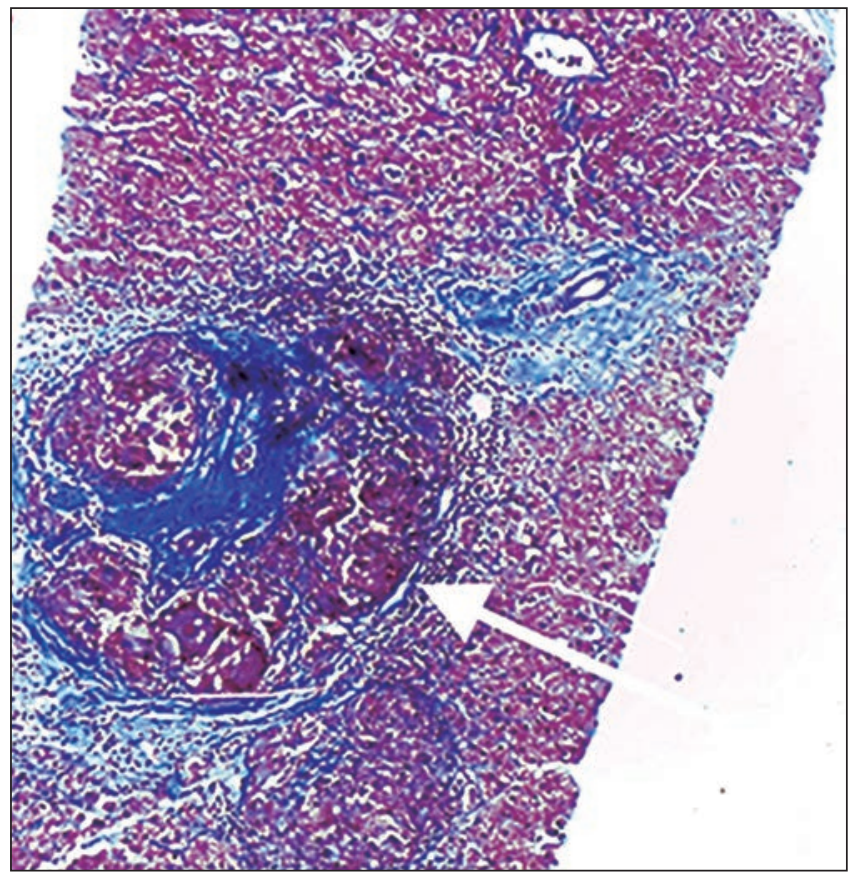

menor impacto en los niveles de glucemia. Para evaluar el compromiso de esta enfermedad en diferentes órganos se realizó evaluación oftalmológica y cardiológica, que fueron normales. El ecocardiograma Doppler no mostró alteraciones. En la endoscopía digestiva alta se evidenció en esófago, tercio inferior, dos cordones tortuosos mayores a $5 \mathrm{~mm}$, y en estómago gastropatía hipertensiva leve. La paciente continuó en tratamiento con deflazacort $50 \mathrm{mg}$ diarios y controles en su lugar de origen. A los 12 meses presentó HDA con descompensación hemodinámica y falleció.

\section{Discusión}

La etiología de los granulomas hepáticos puede ser de origen infeccioso, inmunológico, neoplásico o secundario a drogas. En los países desarrollados predominan las causas no infecciosas, aunque actualmente con la identificación del virus de la hepatitis $\mathrm{C}$ como potencial causa de granulomas y la formación de granulomas por agentes oportunistas en pacientes con diagnóstico de HIV está aumentando el diagnóstico de causas infecciosas a nivel mundial. Sin embargo, se mantiene la tendencia a favor de las patologías inmunológicas en países desarrollados, a diferencia de los países no desarrollados donde predomina la tuberculosis como principal responsable. En el 11\% de los casos no se logra identificar la etiología. ${ }^{1-4}$

Dentro de las causas inmunológicas, la más frecuente es la CBP con una prevalencia de $48,64 \%$, y en segundo 
lugar la sarcoidosis hepática con $8,37 \% .^{5}$ En nuestra paciente se descartaron causas infecciosas, tales como TBC, hepatitis C, B y HIV. No refería consumir drogas ni había datos clínicos que sugirieran patología tumoral. No presentaba anticuerpos reactivos para CBP. Teniendo en cuenta el género de la paciente y habiendo descartado otras etiologías probables, se arribó al diagnóstico de sarcoidosis hepática, apoyados por la anatomía patológica y el dosaje de ECA.

La sarcoidosis es una enfermedad multisistémica. El compromiso intra y extratorácico simultáneos ocurren en un $40-50 \%$, mientras que su afectación extratorácica única ocurre en menos del 10\%. El compromiso hepático es frecuente, pero su incidencia en la práctica clínica está desestimada ya que suele presentarse de manera asintomática. ${ }^{6,7} \mathrm{Su}$ porcentaje de presentación en autopsias viene en aumento y oscila entre $50-80 \% .^{3}$ Solo un $5 \%$ a $15 \%$ presentan dolor abdominal, $5 \%$ presentan signos de síndrome constitucional tales como pérdida de peso, sudoración nocturna y fiebre, de 15 a $40 \%$ presentan hepatoesplenomegalia, y un 20 a 40\% presentan alteración de las enzimas hepáticas. ${ }^{8}$

El compromiso hepático de la enfermedad ha sido reportado con mayor frecuencia en personas de origen europeo. El primer reporte de sarcoidosis hepática asociado a hipertensión portal fue publicado en el año 1949 por Mino. ${ }^{9}$ El 3 a 18\% de los casos con evolución natural de la enfermedad por un período largo presentan HTP secundaria. La fisiopatología es discutida. Algunas teorías sugieren pequeños shunts arterio-venosos en la región de los granulomas o secundario a la compresión de la vena porta por parte de estos. ${ }^{3,6} \mathrm{La}$ forma clásica de presentación es una lesión granulomatosa, pero no es la única lesión histológica. En algunos casos, sobre todo en pacientes afroamericanos y jamaiquinos, se presenta como una colestasis intrahepática, también llamada colangitis granulomatosa, y que se asemeja a una CBP excepto por los granulomas. También se han encontrados casos de cirrosis, secundario a patologías hepáticas sobreagregadas o secundario a la larga evolución de la colestasis. ${ }^{8}$ Sin embargo, la presentación con hipertensión portal y cirrosis es rara; es menor al $1 \%$ y conlleva un peor pronóstico. ${ }^{6}$

En nuestra paciente esta fue la forma de presentación de la enfermedad.

Por laboratorio, la ECA, que es producida por las células en el granuloma, se encuentra elevada en el $60 \%$ de los casos, y es significativamente más elevada en los casos de la enfermedad con compromiso extratorácico. Tiene una sensibilidad de 70,6\% y especificidad de $80 \%$. Por presentar baja sensibilidad, un valor negativo de la misma no descarta la enfermedad. ${ }^{10}$ En nuestra paciente la enzi- ma se encontraba elevada, apoyando nuestro diagnóstico, y en concordancia con el $60 \%$ reportado en la literatura.

Si bien no se cuenta con un método por imágenes con una alta sensibilidad para la detección de granulomas hepáticos, la ecografía, la tomografía y la resonancia con y sin gadolinio son los métodos de mayor uso, siendo esta última una herramienta recomendada para el estudio de la sarcoidosis con compromiso abdominal. ${ }^{6}$ No se recomienda el uso del PET Scan en forma rutinaria para evaluar el compromiso hepático de esta patología, ya que el hepatocito normal capta el FDG-glucosa.

El tratamiento de la sarcoidosis hepática es actualmente un tema de debate, y aún no existe una terapéutica específica. Está basado en el estatus del paciente y en el grado de compromiso de la enfermedad. En los casos leves asintomáticos, se cree que estos recuperan su función espontáneamente, mientras que en aquellos que presentan alteraciones basadas en parámetros bioquímicos, tales como colestasis o sintomatología clínica, se han probado múltiples tratamientos. Dado el efecto antiinflamatorio de los corticoides, se han realizado estudios con monoterapia en pacientes con alteraciones de las enzimas hepáticas, que han evidenciado respuesta completa en un tercio de los pacientes, $50 \%$ de respuesta en otro tercio y un tercio que no obtiene respuesta. ${ }^{5}$ Considerando los efectos adversos de la corticoterapia sistémica y la falta de evidencia de que cambien el curso natural de la enfermedad, se recomienda su uso únicamente ante grandes elevaciones de las enzimas hepáticas, HTP o descompensación hepática en estadios avanzados. El uso de budesonide vía oral es una terapéutica atractiva dado su efecto antiinflamatorio, metabolismo y gran concentración hepática y pocos efectos sistémicos, aunque no hay estudios publicados sobre su uso en sarcoidosis hepática. ${ }^{7}$ El tratamiento con ácido ursodesoxicólico mejora los síntomas de colestasis y en algunos reportes de caso demostró evitar la progresión de la enfermedad. ${ }^{11-13}$ Sin embargo, el uso de grandes dosis de esta droga se vio asociada a mayor mortalidad en pacientes con colestasis secundaria a CBP, por lo que no se recomienda su uso de forma rutinaria. ${ }^{7}$ Existen algunos trabajos con azatioprina, metotrexato y talidomida en los que se evidencia mejoría de los síntomas y de los valores de las enzimas hepáticas, pero aún hay poca evidencia e indicación precisa para su uso. ${ }^{7}$ Se ha estudiado el uso de anticuerpos monoclonales, principalmente infliximab, en la sarcoidosis extratorácica, que ha mostrado buenos resultados en asociación con prednisona para los casos con compromiso hepático refractario. ${ }^{7,14,15}$ Son raros los casos en los que existe insuficiencia hepática severa, por lo que la experiencia con el trasplante hepático es limitada. En un trabajo retrospectivo de un mismo centro, se lleva- 
ron a cabo 13 casos de trasplante hepático a pacientes con sarcoidosis hepática. De estos, cuatro $(30,76 \%)$ tuvieron recaída de la enfermedad de base. En este estudio se hallaron peores resultados en el período postrasplante, tanto en el injerto como en la sobrevida de los pacientes, en comparación con otras enfermedades colestásicas que requirieron trasplante, como $\mathrm{CBP}$, con una supervivencia de $84,6 \%, 76,9 \%, 61,1 \%$ y $51,3 \%$ al primer, tercer, quinto y décimo año. ${ }^{16-18}$ La explicación de estos resultados podría deberse a que la enfermedad es multisistémica. En cuanto al régimen de inmunosupresión postrasplante en este estudio, se utilizaron corticoides, junto con micofenolato y tacrolimus al principio, suspendiendo luego los corticoides. En estos pacientes no se objetivó descompensación de la enfermedad en un plazo a 10 años. ${ }^{18}$

En nuestro caso, decidimos iniciar monoterapia corticoide, con deflazacort $1 \mathrm{mg}$ por $\mathrm{kg}$ de peso por día y evaluar la respuesta terapéutica. La paciente continuó su seguimiento en su ciudad natal y falleció a causa de HDA al año de la internación.

\section{Conclusión}

La sarcoidosis sistémica con compromiso hepático, por lo general, se presenta de manera asintomática, por lo que una alta sospecha clínica nos permitirá realizar un diagnóstico precoz de la enfermedad, tratando de evitar así las complicaciones que esta enfermedad desarrolla durante su evolución.

\section{Sostén financiero. No obtuvimos soporte financiero.}

\section{Aviso de derechos de autor}

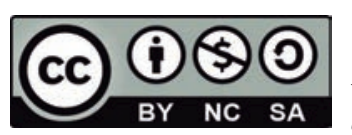

(C) 2021 Acta Gastroenterológica Latinoamericana. Este es un artículo de acceso abierto publicado bajo los términos de la Licencia Creative Commons Attribution (CC BY-NC-SA 4.0), la cual permite el uso, la distribución y la reproducción de forma no comercial, siempre que se cite al autor y la fuente original.

Cite este artículo como: Rivarola SG, Veltri MN, Saguier Padilla I y col. Hipertensión portal secundaria a sarcoidosis hepática. Acta Gastroenterol Latinoam. 2020;50(1):45-9. https://doi.org/10.52787/tdlw1361

\section{Referencias}

1. Geramizadeh B, Jahangiri R, Moradi E. Causes of hepatic granuloma: A 12-year single center experience from southern Iran. Arch Iran Med 2011; 14 (4): 288-289.
2. Gaya DR, Thorburn D, Oien KA, Morris AJ, Stanley AJ. Hepatic granulomas: a 10 year single centre experience. J Clin Pathol 2003; 56 (11): 850-853.

3. Almadi MA, Aljebreen AM, Sanai FM, Marcus V, Almeghaiseeb ES, Ghosh S. New insights into gastrointestinal and hepatic granulomatous disorders. Nat Rev Gastroenterol Hepatol 2011; 8 (8): 455-466.

4. Wainwright H. Hepatic granulomas. Eur J Gastroenterol Hepatol 2007; 19 (2): 93-95.

5. Denk H, Scheuer PJ, Baptista A, Bianchi L, Callea F, De Groote $\mathrm{J}$, et al. Guidelines for the diagnosis and interpretation of hepatic granulomas. Histopathology 1994; 25 (3): 209-218.

6. Tan CB, Rashid S, Dhyan R, Gebre W, Mustacchia P. Hepatic Sarcoidosis Presenting as Portal Hypertension and Liver Cirrhosis: Case Report and Review of the Literature. Case Rep Gastroenterol 2012; 6: 183-189.

7. Jamak M, Esfeh,D, Culver,T, Plesec, BJ. Clinical presentation and protocol for management of hepatic sarcoidosis. Expert Review of Gastroenterology \& Hepatology 2015; 9: 349-358.

8. Karagiannidis A, Karavalaki M, Koulaouzidis A, Ed VC. Artemisa Hepatic sarcoidosis. Ann Hepatol 2006; 5 (4): 4-9.

9. Mino RA, Murphy AI Jr, Livingstone RG. Sarcoidosis Producing Portal Hypertension: Treatment by Splenectomy and Splenorenal Shunt. Ann Surg 1949; 130 (5): 951-957.

10. Yasar Z, Özgül MA, Cetinkaya E, Kargi A, Gül, Talay F, Tanriverdi E, Dincer HE. Angiotensin-converting Enzyme as a Predictor of Extrathoracic Involvement of Sarcoidosis. Sarcoidosis Vasc Diffus Lung Dis 2016; 32 (4): 318-324.

11. Tan CB, Rashid S, Rajan D, Gebre W, Mustacchia P. Hepatic Sarcoidosis Presenting as Portal Hypertension and Liver Cirrhosis: Case Report and Review of the Literature. Case Rep Gastroenterol. 2012; 6 (1): 183-189.

12. Ratziu V, de Ledinghen V, Oberti F, Mathurin P, Wartelle-Bladou C, Renou C, et al. A randomized controlled trial of highdose ursodesoxycholic acid for nonalcoholic steatohepatitis. J Hepatol 2011; 54 (5): 1011-1019.

13. Cremers JP, Drent M, Baughman RP, Wijnen PA, Koek GH. Therapeutic approach of hepatic sarcoidosis. Curr Opin Pulm Med 2012; 18 (5): 472-482.

14. Callejas-Rubio JL, López-Pérez L, Ortego-Centeno N. Tumor necrosis factor-alpha inhibitor treatment for sarcoidosis. Ther Clin Risk Manag 2008; 4 (6): 1305-1313.

15. Kennedy PTF, Zakaria N, Modawi SB, Papadopoulou AM, Murray-Lyon I, du Bois RM, et al. Natural history of hepatic sarcoidosis and its response to treatment. Eur J Gastroenterol Hepatol. 2006; 18 (7): 721-726.

16. Maddrey WC, Johns CJ, Boitnott JK, Iber FL. Sarcoidosis and chronic hepatic disease: a clinical and pathologic study of 20 patients. Medicine (Baltimore). 1970; 49 (5): 375-395.

17. Vanatta JM, Modanlou KA, Dean AG, Nezakatgoo N, Campos L, Nair S, et al. Outcomes of orthotopic liver transplantation for hepatic sarcoidosis: an analysis of the United Network for Organ Sharing/Organ Procurement and Transplantation Network data files for a comparative study with cholestatic liver diseases. Liver Transpl. 2011; 17 (9): 1027-1034.

18. Muhammad B, Sanjaya K, Satapathy Y, Mohammad KI, Jason MV. Long-Term Outcomes of Liver Transplantation for Hepatic Sarcoidosis: A Single Center Experience Journal of Clinical and Experimental. Hepatology 2016; 6: 94-99. 